\title{
The Trimming-Based Design Method for PWR Coolant Flow Distribution Device
}

\author{
Qian Hui $\mathbb{D}^{1},{ }^{1}$ Wenqiang Li $\mathbb{D},{ }^{1}$ Yan Li $\mathbb{D},{ }^{1}$ Zhonghui Wang, $^{2} \mathrm{Hao} \mathrm{Li}^{2}$ and Xin Guo $\mathbb{D}^{1}$ \\ ${ }^{1}$ School of Manufacturing Science \& Engineering, Sichuan University, Chengdu 610065, China \\ ${ }^{2}$ Nuclear Power Institute of China, Chengdu 610065, China
}

Correspondence should be addressed to Wenqiang Li; liwenqiang@scu.edu.cn

Received 14 February 2019; Accepted 1 April 2019; Published 2 May 2019

Academic Editor: Eugenijus Ušpuras

Copyright (C) 2019 Qian Hui et al. This is an open access article distributed under the Creative Commons Attribution License, which permits unrestricted use, distribution, and reproduction in any medium, provided the original work is properly cited.

In order to resolve the situations of nonuniform coolant flow distribution and insufficient vortex suppression, the existing Pressurized Water Reactor (PWR) usually adopts complex coolant mixing structures. However, those structures will greatly increase the complexity and maintenance cost of the system. To solve this problem, a trimming-based design method is proposed in this paper for the complex system and applies it to the design process of the PWR coolant flow distribution device. The function model of the coolant flow distribution system is built based on its functional analysis, and, according to the result of the component feature analysis, the columns and part of the basket are trimmed in order to simplify the overall structure of the system. To further solve the technical contradictions occurred in the simplified system, the contradiction solving tools of TRIZ theory are adopted. By setting the stereo flow equalizing plate, which can strengthen the function of flow distribution and vortex suppression, a coolant flow distribution device for PWR based on dome structure is obtained finally. This device owns a simple structure with good effect on coolant flow distribution and vortex suppression, which can achieve the goal of uniform coolant flow distribution of the system effectively.

\section{Introduction}

With the development of science and technology and the increasing shortage of energy in the world, more and more countries are attaching importance to nuclear energy as a clean energy [1]. Meanwhile, countries have increasingly high requirements on the safety of nuclear energy [2-4].

PWR is the most mature reactor type of the current technology. It contains components like the reactor pressure vessel, the internal components, and the core. The coolant flow channel is composed of the reactor internals, the inner wall of the pressure vessel, and the fuel assembly structure. The coolant would then pass through the passing loop to draw the thermal energy generated by nuclear fission from the core. The distribution uniformity of reactor coolant in the core will directly affect the thermal and hydraulic behavior in the core and then affect the operating limit of the nuclear power plant. In addition, uneven flow distribution will also lead to insufficient core cooling. The large local temperature change in the reactor core will bring hidden trouble to the safe operation of the reactor. Thus, the uniform distribution of coolant in the core is of great significance to the safety and economy of a nuclear reactor $[5,6]$.

There are two main reasons for the uneven distribution of coolant flow in PWR.

Firstly, according to the structural characteristics of the PWR and the flow characteristics of the fluid in the reactor, the coolant has a tendency to fall along the inner wall of the lower plenum at the bottom of the reactor pressure vessel and flow to the center of the lower plenum. As a result, more coolant flow is distributed at the center than the edge of the core inlet.

Secondly, since the lower head of the pressure vessel is mostly spherical, a large number of vortexes will be generated in the lower plenum due to the sharp change of the flow passage and the large depth of the hemispherical head when the coolant flows into the lower plenum from the inlet nozzle of the pressure vessel. The vortexes generated will consume a lot of energy and further cause the distribution of the coolant to be uneven. Due to the role of vortex, the components in 
the lower plenum will vibrate, which will affect the stability of the structure and cause potential safety hazards.

Thus, to ensure the uniform coolant flow distribution in the core, the coolant should be distributed evenly before entering the core. During the distribution process, the generation of vortex should be minimized so as to reduce the coolant pressure drop and the vibration in the lower plenum. To achieve these goals, the existing coolant flow distribution devices are designed as various mating parts in the lower core support plate, mostly like the columns and basket [2, $7,8]$. A large number of columns are bolted to the lower support plate to provide mixing and supporting functions. Due to the complicated structures and assembly relationships of these columns, the cost and maintenance difficulty of the flow distribution devices get increased. Apart from that, there are still some deficiencies of the existing coolant flow distribution devices, such as nonuniform flow distribution, excessive pressure drop, and incomplete vortex suppression.

In the design of the coolant flow distribution device, the designer generally adopted numerical simulation methods to analyze the flow distribution at the core inlet and its influence factors, so as to provide references for the subsequent optimization of the devices [6]. Jeong and Han [5] adopted a commercial computational fluid dynamics (CFD) code, STAR-CD, to analyze the flow distribution and pressure drop in the downcomer and lower plenum of Korean standard nuclear power plants (KSNPs). The real geometry of the KSNPs was used in the analysis. The results presented a clear figure about flow fields in the downcomer and lower plenum of the PWR and provided references for the further research and optimization. Zhang et al. [2] used CFD to calculate the flow field in the reactor lower plenum and put forward optimization design of the existing reactor lower plenum. Yang et al. [9] took the numerical simulation software FLUENT as the tool to research the hydraulic characteristics of the lower plenum of the Qinshan Phase Two PWR. The flow field, pressure field, and flow distribution at the outlet of the lower plenum were obtained to provide valuable references for the design, optimization, and operation management of power stations. Zhang et al. [10] used ANSYS CFX12.1 software to analyze the flow field inside the PWR pressure vessel and obtain parameters of the pressure drop and core inlet flow distribution, etc. By optimizing the structural parameters, a flow distribution structure was finally improved and determined. Sun et al. [11] used CFD to investigate the coolant flow distribution by the effect of a flow mixing chamber (FMC) and analyze velocity and temperature characteristics under different low power conditions. An optimized FMC configuration has been proposed, which can help to improve the nonuniform coolant temperature distribution at the core inlet effectively.

Although the numerical simulation methods can optimize the existing coolant flow distribution devices, the designers are limited by their own domain knowledge and professional experience. And as the designers are lacking innovative design methods and corresponding knowledge resources, they are difficult to jump outside of the box to propose innovative design of the coolant flow distribution device.
According to the causes of nonuniform coolant flow distribution in PWR and considering the shortcomings and design bottlenecks of the existing products, a trimmingbased design method for PWR coolant flow distribution device and its design flow are proposed in this paper. In order to solve the contradictions in design and generate satisfying solutions accurately and quickly, TRIZ theory and its tools are adopted. By building the trimming-based design process model and using innovative design methods and tools such as functional analysis, trimming, and contradiction matrix, a PWR coolant flow distribution device based on dome structure is presented here. This device can overcome the shortcomings of the existing technology and owns a simple structure with good effect on coolant flow distribution and vortex suppression, which achieves the goal of uniform coolant flow distribution of the system effectively.

\section{The Trimming-Based Innovative Design Method}

Trimming is an analytical and problem-solving method of TRIZ theory. Its essence is to remove the component(s) and then reassign its useful function to others in the system or super system to eliminate conflicts or reduce costs [12-14]. Trimming provides effective support for innovative design of complex systems and it improves the system functions by reducing its components [15]. There are two main issues being concerned about during the trimming process. One is the determination of the component(s) to be trimmed, and the other is to improve the functions of the trimmed system.

The determination of the components to be trimmed and their trimming priority is made based on the function model of the system. There are two principles being recommended for determination of the component trimming priority, i.e., "most critical key disadvantage" and "most expensive components" $[12,16]$. Cause effect chain analysis (CECA) or cause effect contradiction chain analysis (CECCA) can be used to identify the most critical key disadvantages $[17,18]$, which include harmful functions, excessive functions, and insufficient functions in the engineering system. Usually, the harmful functions are the priority target of elimination [19]. Another way to determine the component trimming priority is based on the cost of each component. Naturally, the higher the component costs, the higher the priority to be trimmed.

To improve the functions of the trimmed system, functional analysis is carried out in the trimmed system. Then the trimming problems, which are the new problems or contradictions of the system, are identified and resolved by the contradiction solving tools of TRIZ. TRIZ is a theory of inventive problem solving proposed by G. S. Altshuler, who believed that the core of the inventive problem solving is to identify and resolve its contradictions [20, 21]. Using TRIZ to stimulate the designers to generate creative solutions can not only increase the success rate of production innovation and shorten the cycle of product development but also make the problems more predictable [22].

Based on the two issues above, this present work establishes a trimming-based innovative design process model as shown in Figure 1. The model contains four main phases, i.e., 


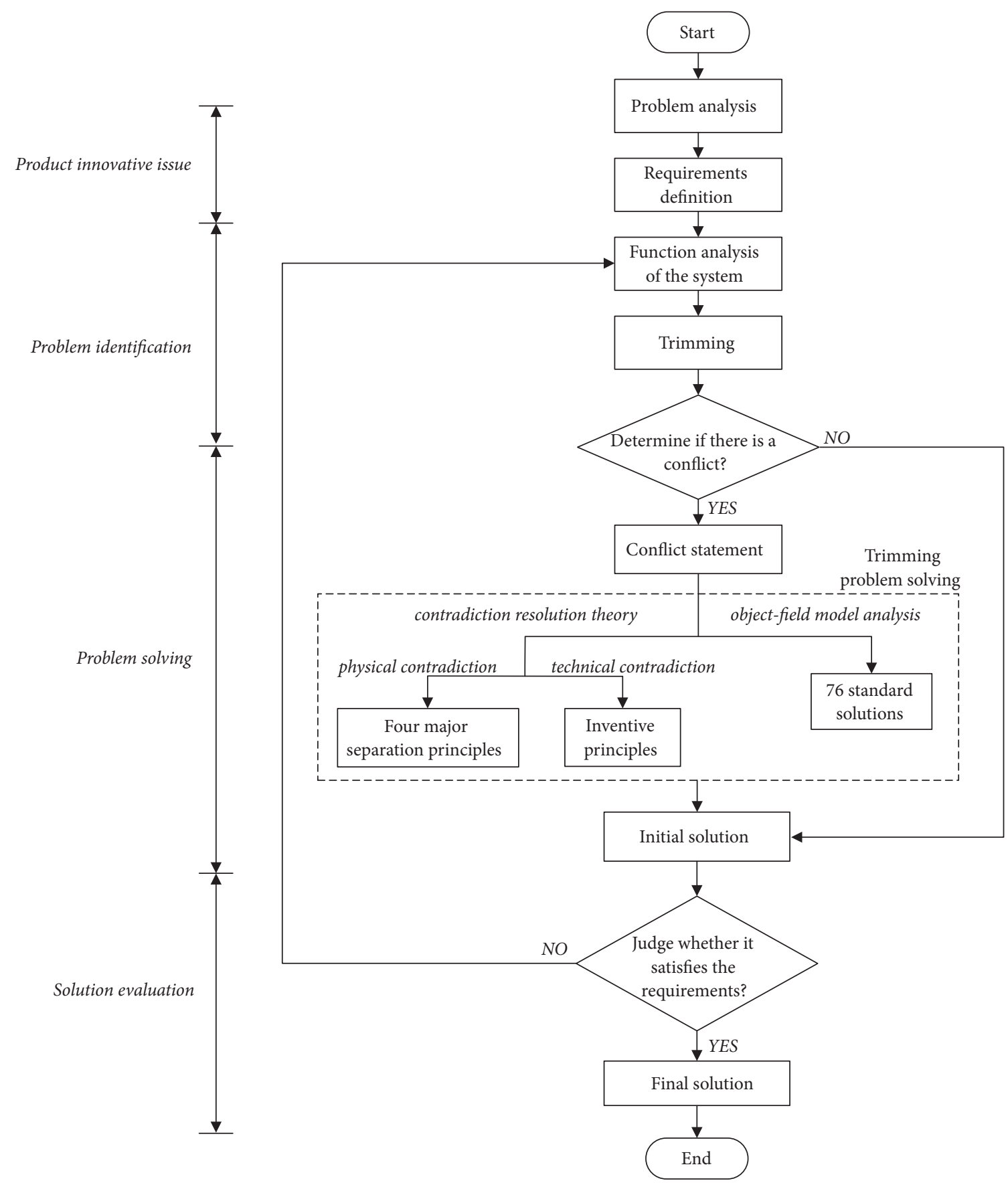

FIGURE 1: The trimming-based innovative design process model.

production innovative issue, problem identification, problem solving, and solution evaluation.

The trimming-based innovative design process can be divided into six steps:

(1) Analyze the design problem and define the design requirements.

(2) Establish the function model based on the functional analysis of the system, and determine the ones to be trimmed according to the characteristics of each component of the system.
(3) Trim the selected components according to the design requirements and build the new function model of the trimmed system.

(4) Analyze the output of the trimming process and formulate the trimming problem. If there is no trimming problem, proceed to step 6; otherwise, move on to the next step.

(5) Identify the contradictions of the simplified system. Apply TRIZ, e.g., inventive principles, standard solutions, separation principles, and effects to solve the 


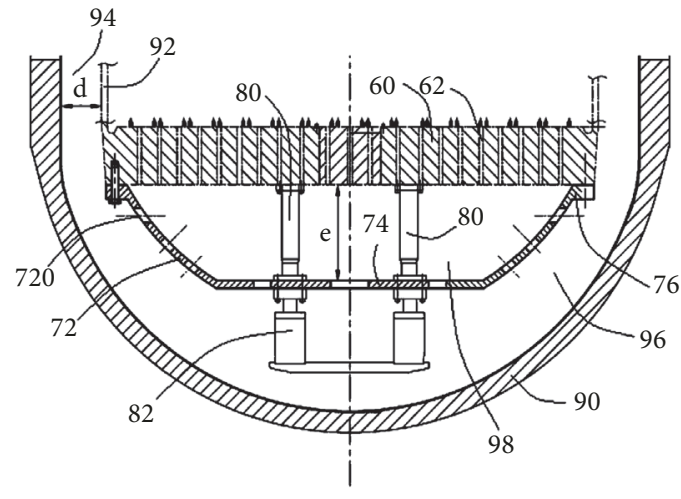

(a) The reactor coolant flow distribution device

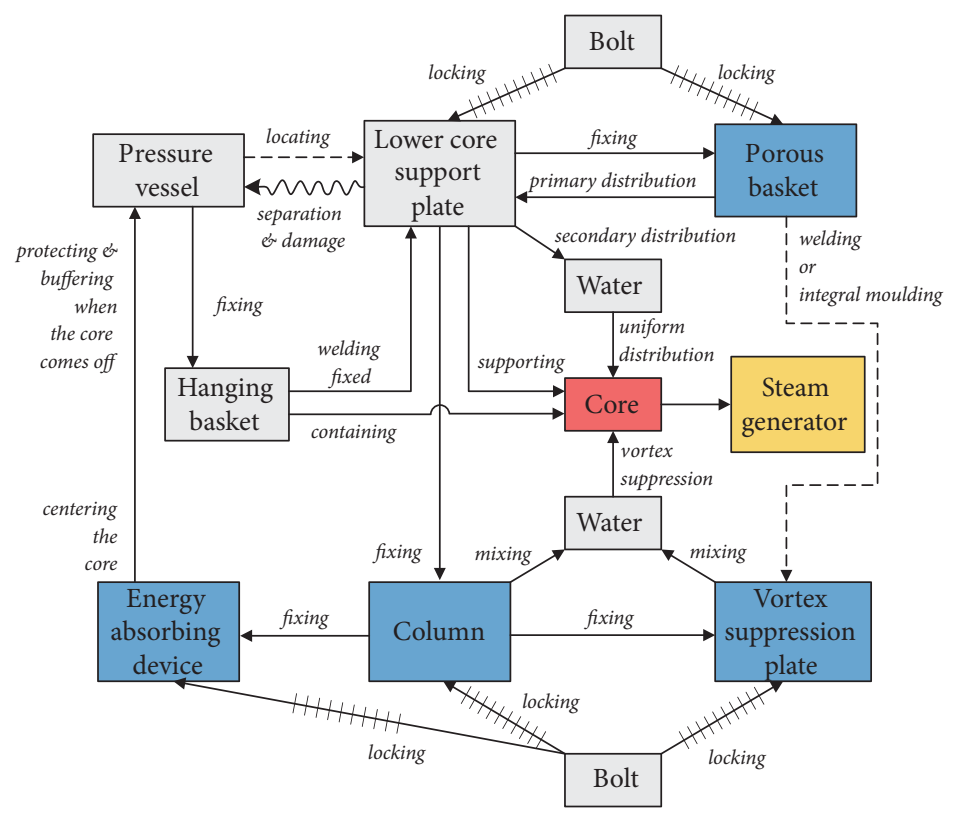

(b) Function analysis

Figure 2: Functional analysis of the coolant flow distribution system. (a) The reactor coolant flow distribution device [23]. (b) Function model.

contradictions to achieve uncompromising design and generate the solution.

(6) Evaluate the solution to determine if it meets the design requirements. If the requirements are met, the design process ends; otherwise, return to step (2) to analyze the system function again and identify the new problem.

\section{The Trimming-Based Design Process for PWR Coolant Flow Distribution Device}

3.1. Product Innovative Issue. According to the trimmingbased design process model shown in Figure 1, the first step to take is to analyze and define the requirements of the PWR coolant flow distribution device. The basic design requirements for the device are the specific functions to be realized, i.e., uniform coolant flow distribution, sufficient coolant mixing, and vortex suppression. Besides, considering the shortcomings of the existing products, it is also required that the device should overcome the disadvantages of the existing technologies and simplify the structure and assembly relationships of the system components, thereby reducing its manufacturing cost and the maintenance difficulty.

\subsection{Problem Identification}

3.2.1. Functional Analysis of the System. A product is usually composed of different components and functional analysis is used to establish the interactive relationships between them [24]. Functional analysis is an important method for product innovative design. It helps the designers to transform the mechanical structure into product functions; thus, the designers will not be bound by the existing structure so as to generate new design ideas. Take the reactor coolant flow distribution device [23], which is illustrated as in Figure 2(a), as an example. Three components are mainly contained in this device, i.e., the lower core support plate (60), the basketshaped structure, and the columns (80). The basket-shaped structure is fixed under the lower core support plate and it comprises a porous basket (72) and a vortex suppression plate (74) at the bottom of it. The columns are vertically disposed between the vortex suppression plate and the lower core support plate. According to the functional analysis, the function model of the coolant flow distribution system is established as shown in Figure 2(b).

3.2.2. Trimming. Regarding the design requirement of "simple structure and assembly relationships", the most critical key disadvantage of the coolant flow distribution system is the structure of the columns and porous basket. To simplify the technical system, these components are trimmed on condition that their useful functions, i.e., the fixing and mixing and distribution functions, are well preserved. By assigning these functions to the new function carrier components, the technical system of the device is more simplified, whose performance remains the same or even better with less cost. In a word, through trimming process, the design of PWR coolant flow distribution device is approaching the final ideal solution.

The trimming process of the PWR coolant flow distribution device and the function model of its trimmed system are shown in Figure 3.

Firstly, the porous basket is trimmed partially as shown in Figure 4. According to the functional analysis, the porous 


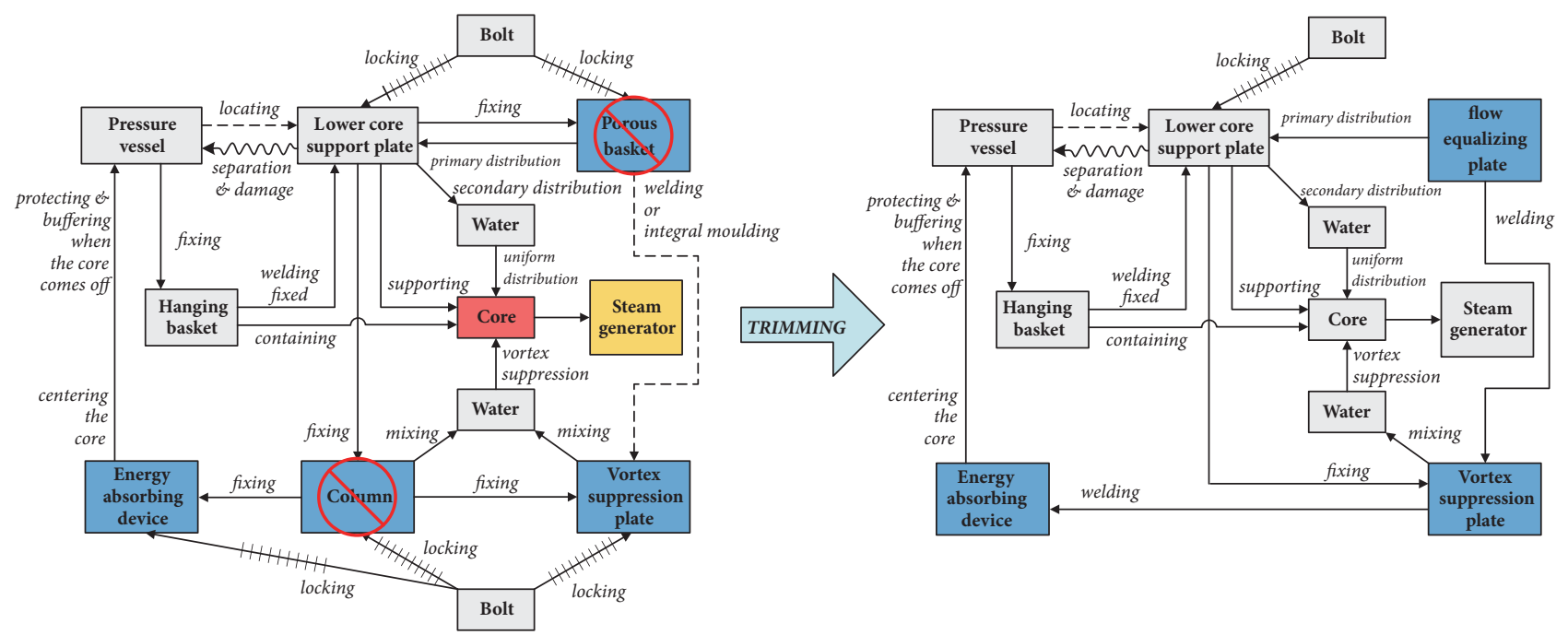

FIgURE 3: Trimming process of the PWR coolant flow distribution device.

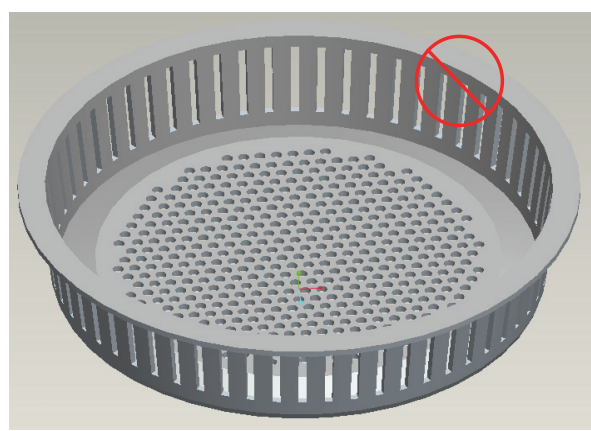

The porous basket

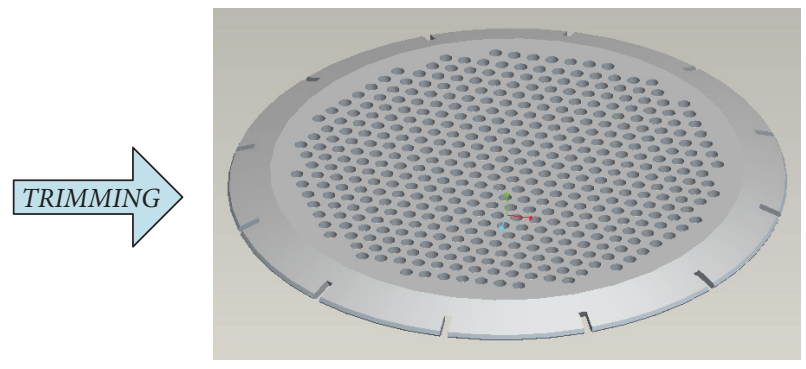

The flow equalizing plate

FIGURE 4: Trimming of the porous basket.

basket is used to distribute the coolant primarily. This function is mainly realized by the bottom flow equalizing plate, while the side wall plays a minor role. Thus, the side wall of the porous basket gets trimmed and the retained flow equalizing plate is connected with the vortex suppression plate by welding. As a result, the connecting bolts are omitted.

Secondly, the columns are trimmed as illustrated in Figure 5. The columns provide both the useful functions (such as "mixing" and "supporting") and harmful functions (complex structure and assembly relationships). Thus while deleting the harmful functions, the useful ones should be kept and reassigned to other components in the system. To be specific, the new function carriers of "mixing" are the vortex suppression plate and the flow equalizing plate. And to realize the "supporting" function, mechanics principles of the dome structure are retrieved and transferred to the current design. With these principles, the vortex suppression plates are inserted into a dome structure as shown in Figure 6. Illustrated in Figure 5, a guide cone is arranged on the outside of each circle of transverse vortex suppression plate, and the taper angles of the cones decrease progressively from bottom to top. With the help of guide cones, the coolant is diverted towards the edge of the core inlet, alleviating the tendency that more coolant flow is distributed at the center of the core inlet than the edge. Moreover, all the vortex suppression plates, including the longitudinal and the transverse ones, are able to cut the coolant flow and block the complete flow line of the vortex. In this case, the vortexes are eliminated.

3.3. Problem Solving. Once the porous basket and columns are trimmed and their functions are reassigned to the new function carrier components, trimming problems will be generated for the corresponding function carriers [25]. These trimming problems will lead to different types of design issues, such as technical contradictions, physical contradictions, or object-field problems [26, 27]. In order to solve the trimming problems, the designer should identify their types firstly and consult TRIZ theory for corresponding tools (see Figure 1) to generate innovative solutions.

It is analyzed that the trimming problem of the trimmed PWR coolant flow distribution device is a technical contradiction. And this practical engineering problem can be resolved by the technical contradiction resolution theory. 


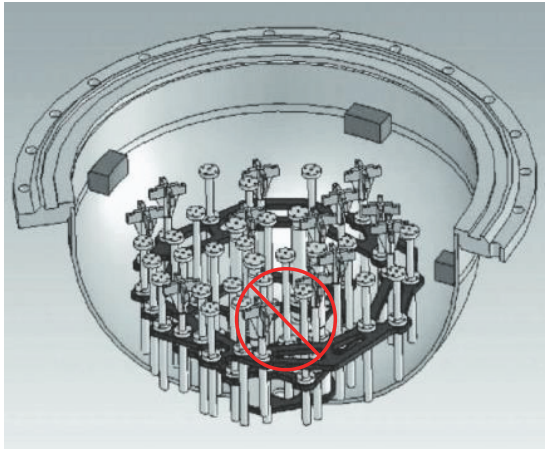

The columns

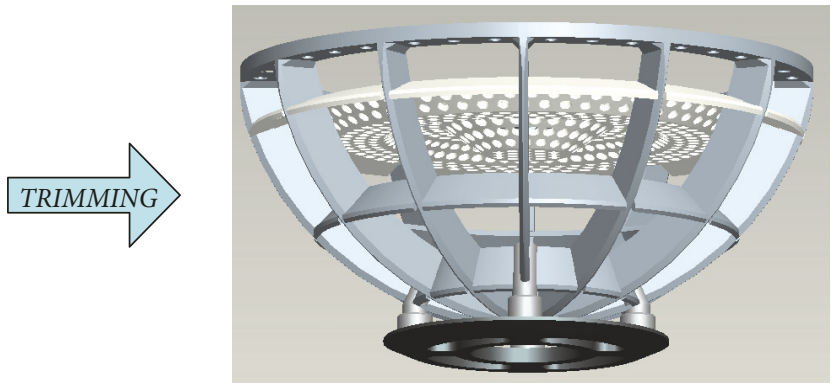

The dome structure

FIgURE 5: Trimming of the columns.

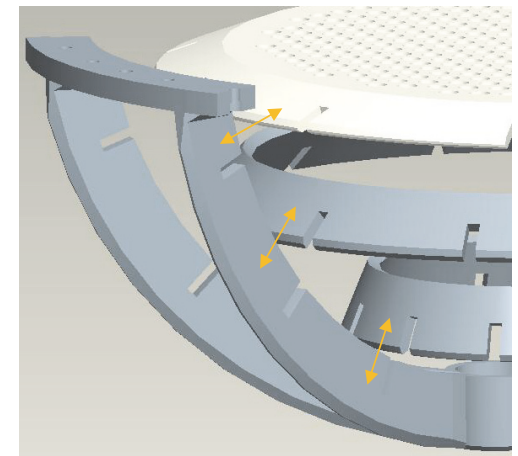

FIGURE 6: Illustration of vortex suppression plates assembly.

Step 1 (define the technical contradiction). While enhancing the mixing and flow distribution effects of the vortex suppression plate and the flow equalizing plate, the pressure drop of the coolant may be increased to cause more vortexes. According to TRIZ, this technical contradiction can be characterized as two of the 39 general parameters, namely, parameter 6 (area of stationary objects) and parameter 22 (energy loss). With these parameters, the contradiction can be represented as a standard TRIZ problem.

Step 2 (query the contradiction matrix). To solve the technical contradiction, the contradiction matrix (refer to Figure 7) is demanded to search for the appropriate inventive principles preliminarily, which are used to obtain the TRIZ solution model. And combining the actual design issue with the working environment of the device, the inventive principle 17 , spatial dimension change, has been chosen for solving this contradiction.

Step 3 (apply the inventive principle). Inspired by the inventive principle 17, a solution is generated by curving the flow equalizing plate to enlarge its spatial dimension. As shown in Figure 8, a stereo flow equalizing plate is designed with a bending surface in its center. There are several flood holes distributed on the stereo flow equalizing plate. Compared with the common ones, the stereo flow equalizing plate has a larger expansion area so that there are more flood holes on per unit area. By enlarging the spatial dimension, the flow distribution area of the plate gets increased, reducing the coolant pressure drop and the structural resistance coefficient. In this way, the problem of generating more vortexes is resolved. The stereo flow equalizing plate provides the coolant with the secondary distribution, which results in more precise result.

3.4. Solution Description and Evaluation. According to the design process above, an innovative solution of the domebased PWR coolant flow distribution device is generated as shown in Figure 9.

As shown in Figure 9(b), the dome-based PWR coolant flow distribution device comprises a pressure vessel (1), a lower core support plate (10), a dome structure (4), a stereo flow equalizing plate (2), and an energy absorbing device (5). The dome structure is located in the lower plenum of the pressure vessel and it is composed of a plurality of plate-shaped longitudinal vortex suppression plates (6) and transverse vortex suppression plates (7) and a flange (3). The longitudinal vortex suppression plates are distributed between the transverse vortex suppression plates and along their circumference. On the outside of each circle of the transverse vortex suppression plate is the guide cone (8), and the taper angles of these cones decrease progressively from bottom to top. The dome structure is connected with the lower core support plate through a flange which is located at its top. The stereo flow equalizing plate is set in the dome structure, with a bending surface in its center and several flood holes spreading all over it. The energy absorbing device is set near the bottom of the lower plenum and gets fixed at the intersection of the transverse and longitudinal vortex suppression plates of the bottom layer. A hanging basket (11) is fixed on the top of the lower core support plate. The core is placed on top of the lower core support plate, and the coolant is injected into the area between the hanging basket and the inner wall of the pressure vessel. As the coolant flows along the inner wall of the pressure vessel, illustrated in Figure 9(b), the transverse and longitudinal vortex suppression plates cut the coolant to block the complete flow line of the vortexes, so as to eliminate the vortexes.

To further verify the effects of coolant flow distribution and vortex suppression of this proposed innovative solution, 


\begin{tabular}{|c|c|c|c|c|c|c|c|}
\hline \multirow{2}{*}{\multicolumn{2}{|c|}{$\begin{array}{l}\text { Improved } \\
\text { general } \\
\text { engineering parameters }\end{array}$}} & No. 1 & No. 2 & $\ldots$ & No. 22 & $\ldots$ & No. 39 \\
\hline & & $\begin{array}{c}\text { Weight of } \\
\text { moving objects }\end{array}$ & $\begin{array}{c}\text { Weight of } \\
\text { stationary objects }\end{array}$ & $\ldots$ & Energy loss & $\ldots$ & Productivity \\
\hline No. 1 & $\begin{array}{c}\text { Weight of } \\
\text { moving objects }\end{array}$ & + & - & $\cdots$ & \begin{tabular}{c|c}
6,2 \\
34, & 19
\end{tabular} & $\ldots$ & $\begin{array}{l}35,3 \\
24,37\end{array}$ \\
\hline No. 2 & $\begin{array}{c}\text { Weight of } \\
\text { stationary objects }\end{array}$ & - & + & $\ldots$ & $\begin{array}{c}18, \\
28,19 \\
15\end{array}$ & $\ldots$ & $\begin{array}{l}1,28 \\
15,35\end{array}$ \\
\hline ... & $\ldots$ & ... & ... & $\ldots$ & 1 & $\ldots$ & $\ldots$ \\
\hline No. 6 & $\begin{array}{c}\text { Area of } \\
\text { stationary objects }\end{array}$ & & $\begin{array}{l}30,2, \\
14,18\end{array}$ & & & $\cdots$ & $\begin{array}{r}2,18 \\
40,4\end{array}$ \\
\hline ... & $\cdots$ & $\cdots$ & $\cdots$ & $\ldots$ & $\ldots$ & $\ldots$ & $\cdots$ \\
\hline No. 39 & Productivity & $\begin{array}{l}35,26 \\
24,37\end{array}$ & $\begin{array}{c}28,27 \\
15,3\end{array}$ & $\cdots$ & $\begin{array}{l}28,10 \\
29,35\end{array}$ & $\cdots$ & + \\
\hline
\end{tabular}

FIgURE 7: The contradiction matrix.

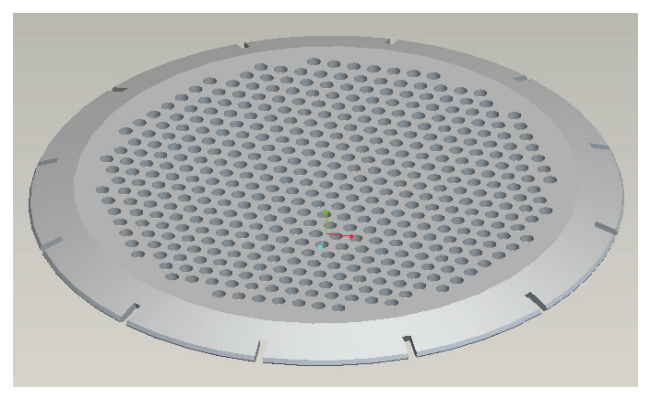

The flow equalizing plate

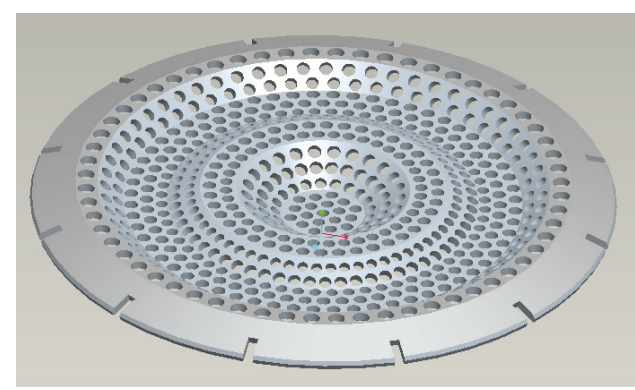

The stereo flow equalizing plate

Figure 8: Curving the flow equalizing plate.

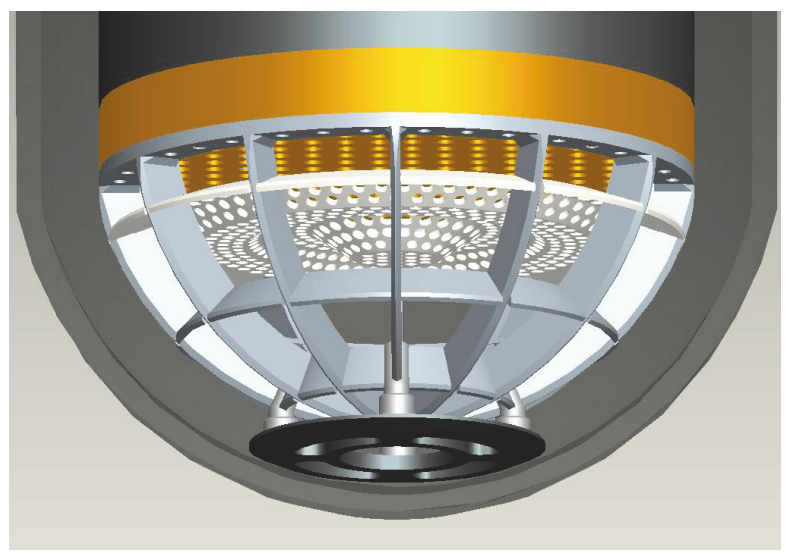

(a) 3D model diagram

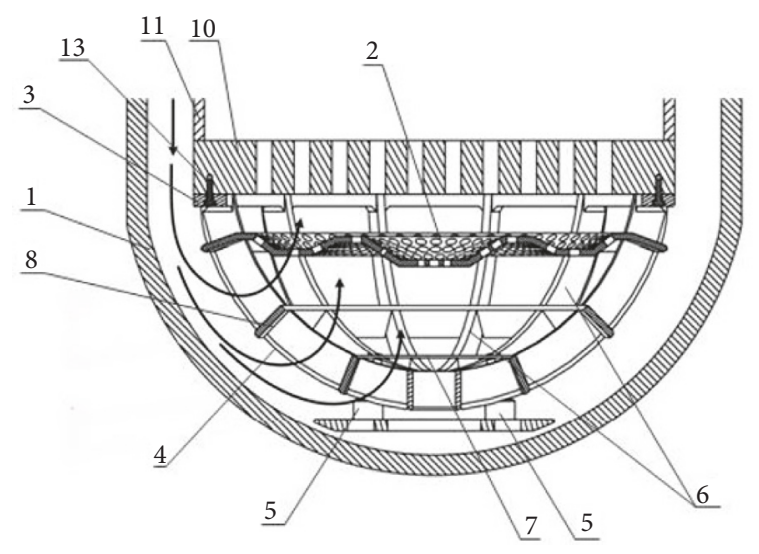

(b) Structure diagram

FIGURE 9: Innovative solution. (a) 3D model diagram. (b) Structure diagram. 
TABLE 1: The parameters and boundary conditions of the simulation model.

\begin{tabular}{lcr}
\hline & The parameters & \\
\hline The turbulence model & & SST model \\
\hline Fluid & & $300^{\circ} \mathrm{C}$ \\
\hline The temperature of the fluid & $15.5 \mathrm{MPa}$ \\
\hline System reference pressure & The boundary conditions & $4.45 \mathrm{~m} / \mathrm{s}$ \\
\hline & & $1.9 \%$ \\
\hline Inlet velocity & $675 \mathrm{~mm}$ \\
\hline Turbulence intensity & $0 \mathrm{~Pa}$ \\
\hline Hydraulic diameter & \\
\hline Outlet pressure (average static pressure)
\end{tabular}

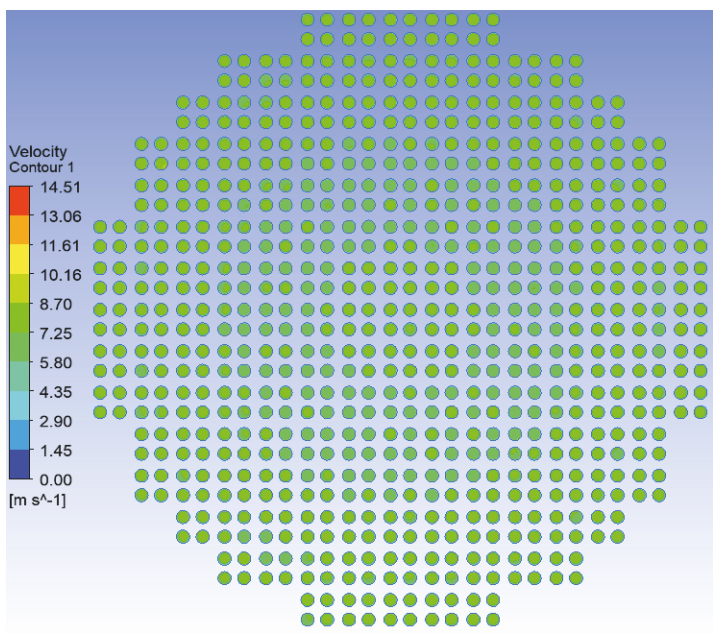

FIGURE 10: Velocity distribution at outlet of the lower core support plate.

Ansys-CFX is used for numerical simulation to calculate the normalized flow at the core inlet and obtain its velocity vector diagram and streamlines diagram. The parameters and boundary conditions of the simulation model are shown in Table 1.

Figure 10 shows the calculation of velocity distribution at outlet of the lower core support plate. It can be preliminarily seen from this figure that the overall velocity distribution of the innovative solution is uniform.

To further describe the characteristics of the core inlet flow distribution, the normalized processing is adopted. The normalized factors of the proposed innovative solution and an existing device [28] are respectively shown in Figures 11(a) and 11(b). The specific data statistics of these two devices and the comparison of their normalized factor proportions are shown in Figure 11(c).

According to Figure 11, the normalized factor of the innovative solution is $0.9<\mathrm{q}<1.08$, while the normalized factor of the existing device is $0.81<\mathrm{q}<1.08$. Therefore, in terms of flow distribution effect, the innovative solution has a better performance than the existing device.

Moreover, the velocity vector diagram and the streamlines diagram of the innovative solution are obtained as illustrated in Figure 12. As the coolant flows into the dome structure, it gets primarily distributed, while the stereo flow equalizing plate provides the secondary distribution for it. During the distribution processes, there exist few small vortexes resulting from the change of the flow direction. But there is no generation of large vortexes and no vortexes remaining in the lower plenum. In general, the proposed innovative solution owns a good vortex suppression effect.

Compared with the existing PWR coolant flow distribution devices, the advantages of the innovative solution here include the following:

(1) The dome structure connects the energy absorbing device and the lower core support plate as a whole, replacing the original columns structure. In this case, fewer screws are used so as to simplify both the assembly relationships and the overall structure of the device.

(2) When the coolant enters the dome structure, it gets primarily distributed. Under pressure, the coolant in the dome structure passes through the flood holes on the stereo flow equalizing plate and enters into the core through the lower core support plate. The stereo flow equalizing plate provides the secondary distribution for the coolant, resulting in more precise result.

(3) The dome structure owns good mechanical properties. In the falling accident of the hanging basket, the dome structure can evenly disperse the impact force and transfer it to the edge of the lower core support plate. Therefore, the original columns can be eliminated and the structure of the device is simplified. Meanwhile, the core flow distribution area of the lower core support plate is avoided to be drilled and equipped with columns, so that the effect of flow distribution gets better.

\section{Conclusion}

In order to improve the coolant flow distribution and supporting functions of the PWR, a trimming-based design method for the complex system is proposed in this paper. The corresponding innovative design process model is established and applied to the specific design of PWR coolant flow distribution device. Considering the shortcomings and design 


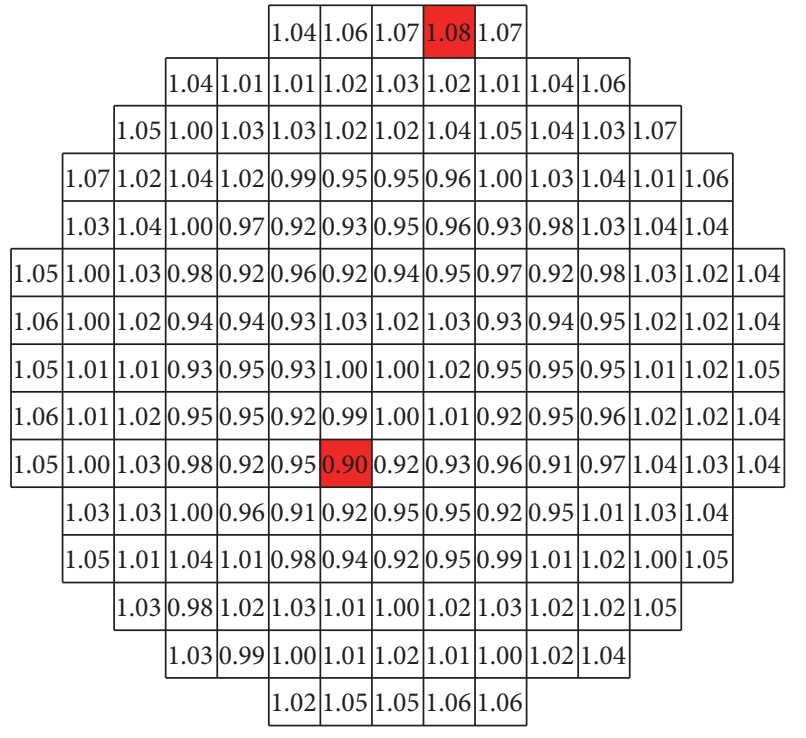

(a)

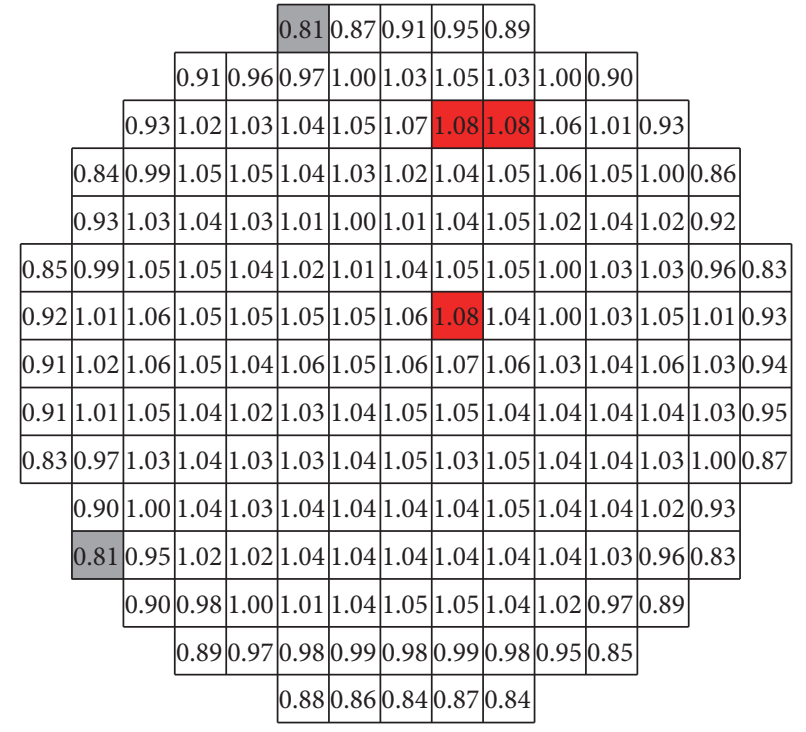

(b)

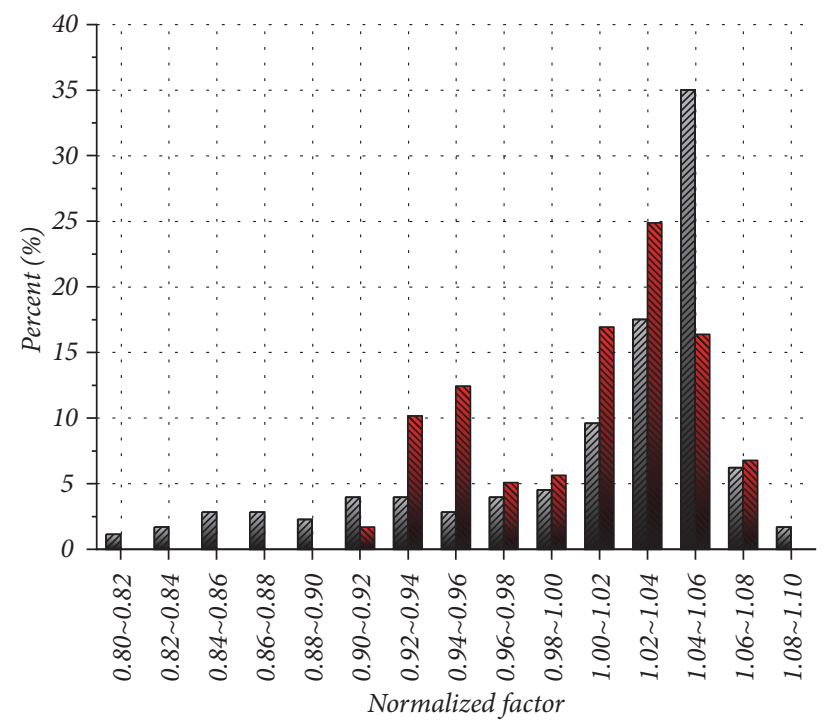

Existing device

Design solution

(c)

FIGURE 11: Normalized factor at the core inlet. (a) Innovative solution. (b) Existing device. (c) Normalized factor proportions comparison.

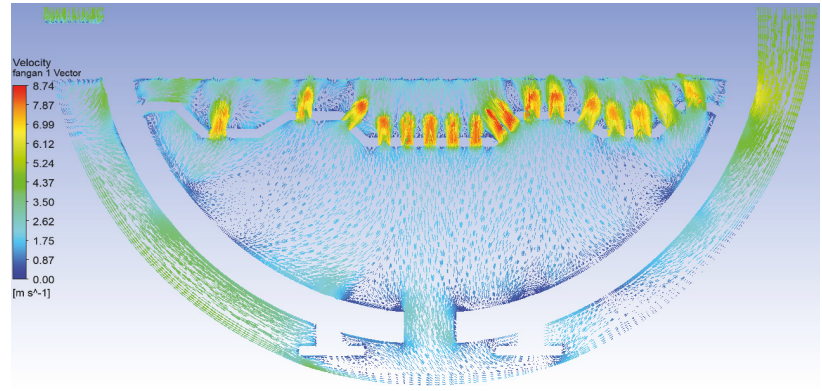

(a)

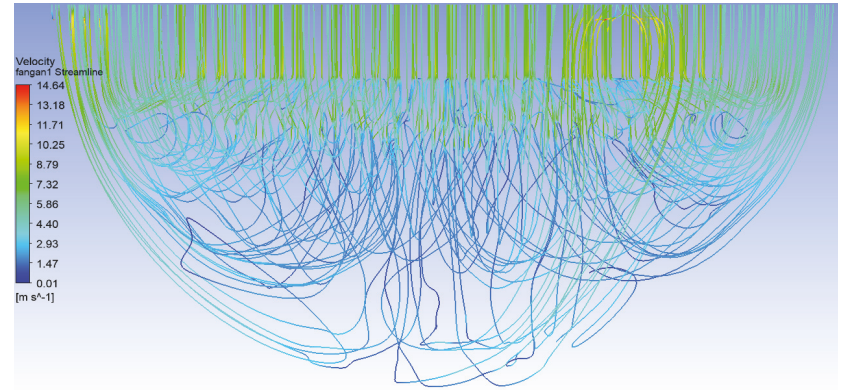

(b)

FiguRE 12: Flow field analysis in the lower plenum. (a) Velocity vector diagram. (b) Streamlines diagram. 
bottlenecks of the existing reactor flow distribution devices, the trimming-based innovative design process model is built with TRIZ theory. The tools of TRIZ are used to solve the contradictions occurred in design. With the help of this model, the designers are supported with innovative design methods and knowledge resources. Different from the existing system optimization methods, the trimmingbased design method achieves function optimization of the system by trimming its components. It can both simplify the structure and assembly relationships of the complex system and provides effective support for the innovative design of the technical system. And for the future work, we will move on to construct an aided tool system based on the design method proposed in this paper to support the trimming-based design for the complex system.

\section{Data Availability}

No data were used to support this study.

\section{Conflicts of Interest}

The authors declare that there are no conflicts of interest regarding the publication of this paper.

\section{Acknowledgments}

The authors would like to express their gratitude to the National Natural Science Foundation of China (No. 51435011), Science \& Technology Ministry Innovation Method Program (No. 2017IM040100), and the Sichuan Applied Foundation Project (No. 2018JY0119).

\section{References}

[1] N. Wang, "Analyzed the safety of nuclear power station," World Scientific Series in R \& D, vol. 24, no. 6, pp. 14-17, 2002.

[2] H. Zhang, H. Liu, C. Fang, and C. Lu, "Optimization design of reactor lower plenum," Nuclear Power Engineering, vol. 35, no. 3, pp. 63-67, 2014 (Chinese).

[3] D. Butler, "Nuclear safety: reactors, residents and risk," Nature, vol. 472, no. 7344, pp. 400-401, 2011.

[4] M. Bunn and O. Heinonen, "Preventing the next fukushima," Science, vol. 333, no. 6049, pp. 1580-1581, 2011.

[5] J. H. Jeong and B.-S. Han, "Coolant flow field in a real geometry of PWR downcomer and lower plenum," Annals of Nuclear Energy, vol. 35, no. 4, pp. 610-619, 2008.

[6] M. Tang, M. Zhang, L. Chen, and X. Yu, "Numerical analysis of flow skirt influence on core inlet flow distribution," Nuclear Power Engineering, vol. 34, no. S2, pp. 83-86, 2013.

[7] Z. Ding, M. Zhang, and S. Lin, "Experiment study on CAP1400 core inlet flow distribution," Atomic Energy Science and Technology, vol. 52, no. 9, pp. 1635-1640, 2018.

[8] Y. Chao, L. Yang, and M. Zhang, "Full vessel CFD analysis on thermal-hydraulic characteristics," Atomic Energy Science and Technology, vol. 48, no. zl, pp. 280-286, 2014.

[9] H. Yang, D. Chen, and Y. Li, "Numerical simulation on hydraulic characteristics of lower plenum of PWR," Journal of
Harbin University of Commerce (Natural Sciences Edition), vol. 30, no. 6, pp. 546-549, 2014.

[10] M. Zhang, Y. Duan, M. Zhu, and J. Pi, “Optimized design for the flow distribution device of nuclear reactor internals based on CFD simulation," Nuclear Science and Engineering, vol. 35, no. 2, pp. 193-199, 2015.

[11] L. Sun, M. Peng, G. Xia, X. Lv, and R. Li, "Numerical study on coolant flow distribution at the core inlet for an integral pressurized water reactor," Nuclear Engineering and Technology, vol. 49, no. 1, pp. 71-81, 2017.

[12] D. D. Sheu and C. T. Hou, "TRIZ-based trimming for processmachine improvements: Slit-valve innovative redesign," Computers \& Industrial Engineering, vol. 66, no. 3, pp. 555-556, 2013.

[13] F. Yu, H. Zhang, J. Sun, R. Tan, and G. Cao, “Trimming-based conflict discovery and problem-solving process model," in Proceedings of the IEEE International Conference on Management of Innovation \& Technology, IEEE, 2012.

[14] D. L. Mann, Hands on Systematic Innovation, UK, 2nd edition, 2007.

[15] D. Xu, TRIZ Innovative Tools, Ding Mao Book Publishing Co., Ltd., China Taibei, 2010.

[16] D. D. Sheu and C. T. Hou, "reakthrough problem solving using TRIZ component trimming," in Proceedings of the 20th International Conference on Industrial Engineering and Engineering Management, Springer, Berlin, Germany, 2013.

[17] D. D. Sheu and M. C. Tsai, "Cause effect and contradiction chain analysis for conflict identification and problem solving," in Proceedings of the 2nd international conference on systematic innovation, New York, NY, USA, 2012.

[18] D. D. Sheu, E. Tsai, and H. Rau, "Integration between causeeffect chain analysis and root contradiction analysis and its applications," in Proceedings of the In the 3rd international conference on systematic innovation, Seoul, Seoul, South Korea, 2012.

[19] R. Tan, Creative Design - TRIZ: Theory of the Inventive Problem Solving, China Machine Press, Beijing, China, 2002.

[20] G. S. Altshuller, The Innovation Algorithm, TRIZ, Systematic Innovation and Technical Creativity, Technical Innovation Center, INC, Worcester, UK, 1998.

[21] R. Tan, Q. Wang, C. Yuan, and G. Duan, "Theory of the inventive problem solving: TRIZ- TRIZ process, tools and," Journal of Machine Design, vol. 18, no. 7, pp. 7-12, 2001.

[22] Y. Li and W. Li, Method to Creative Design, Science Press, Beijingm, China, 2013.

[23] J. Fang, Y. Duan, X. Ran et al., "A flow distribution device in a nuclear power plant reactor," CN Patent No 201410188559.1, 2014.

[24] X. Lu, F. Zhang, and Q. Zhang, "Product innovation design based on the theory of TRIZ and functional analysis," Machinery Design \& Manufacture, vol. 12, pp. 255-257, 2010.

[25] M. Li, X. Ming, L. He, M. Zheng, and Z. Xu, "A TRIZ-based trimming method for patent design around," Computer-Aided Design, vol. 62, pp. 20-30, 2015.

[26] Y. Yang, "TRIZ theory and conflict in the product concept design," Equipment Manufacturing Technolog, vol. 5, pp. 165-166, 2010.

[27] R. Tan, G. Zhang, J. Jiao, and P. Zhang, "Technical contradictions and principles in product design," Journal of Hebei University of Technology, vol. 30, no. 3, pp. 1-6, 2001.

[28] X. Xia, R. Cao, Y. Li et al., "A flow distribution device in the low plenum of a nuclear reactor," CN Patent No 201210540512.8, 2012. 

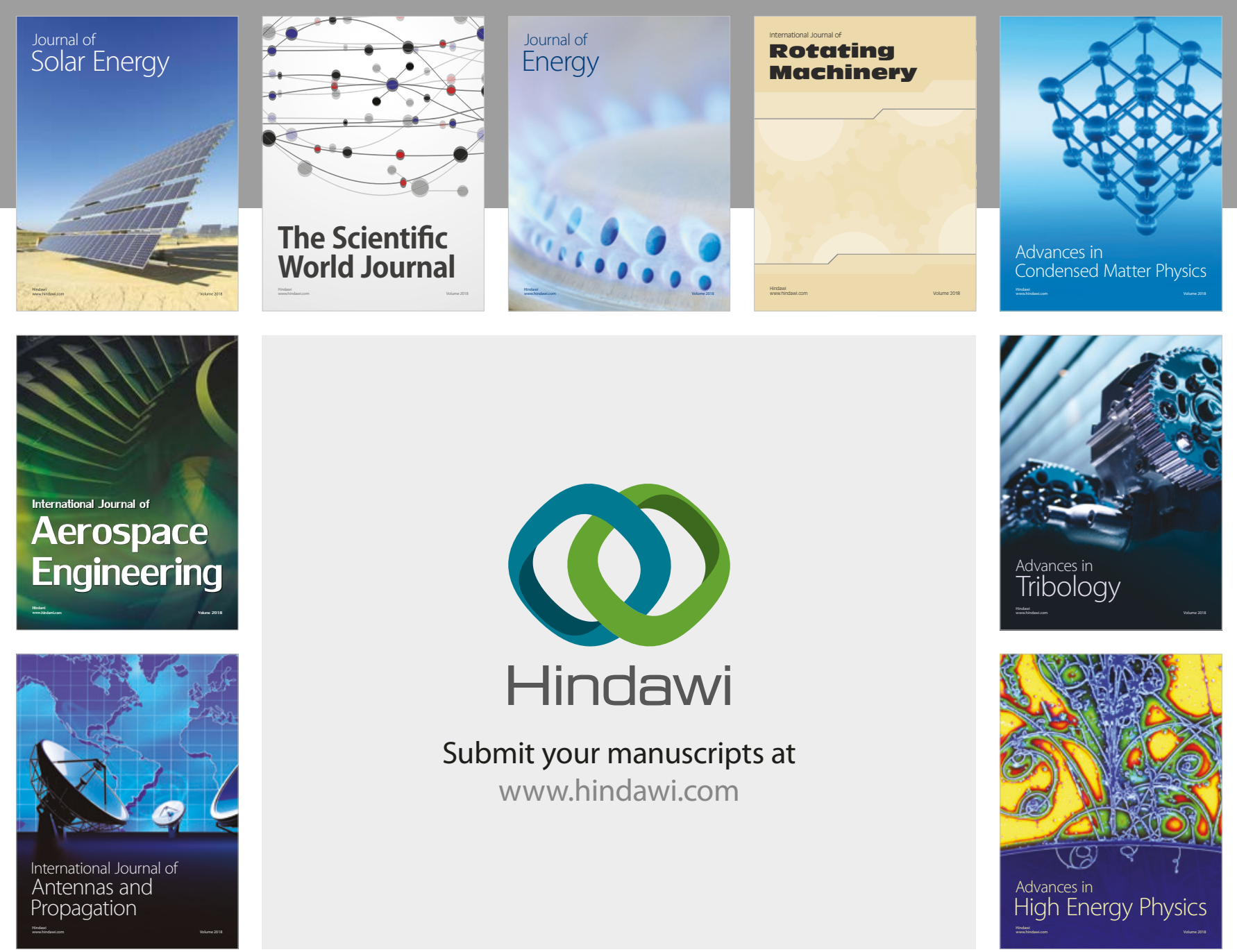

Submit your manuscripts at

www.hindawi.com
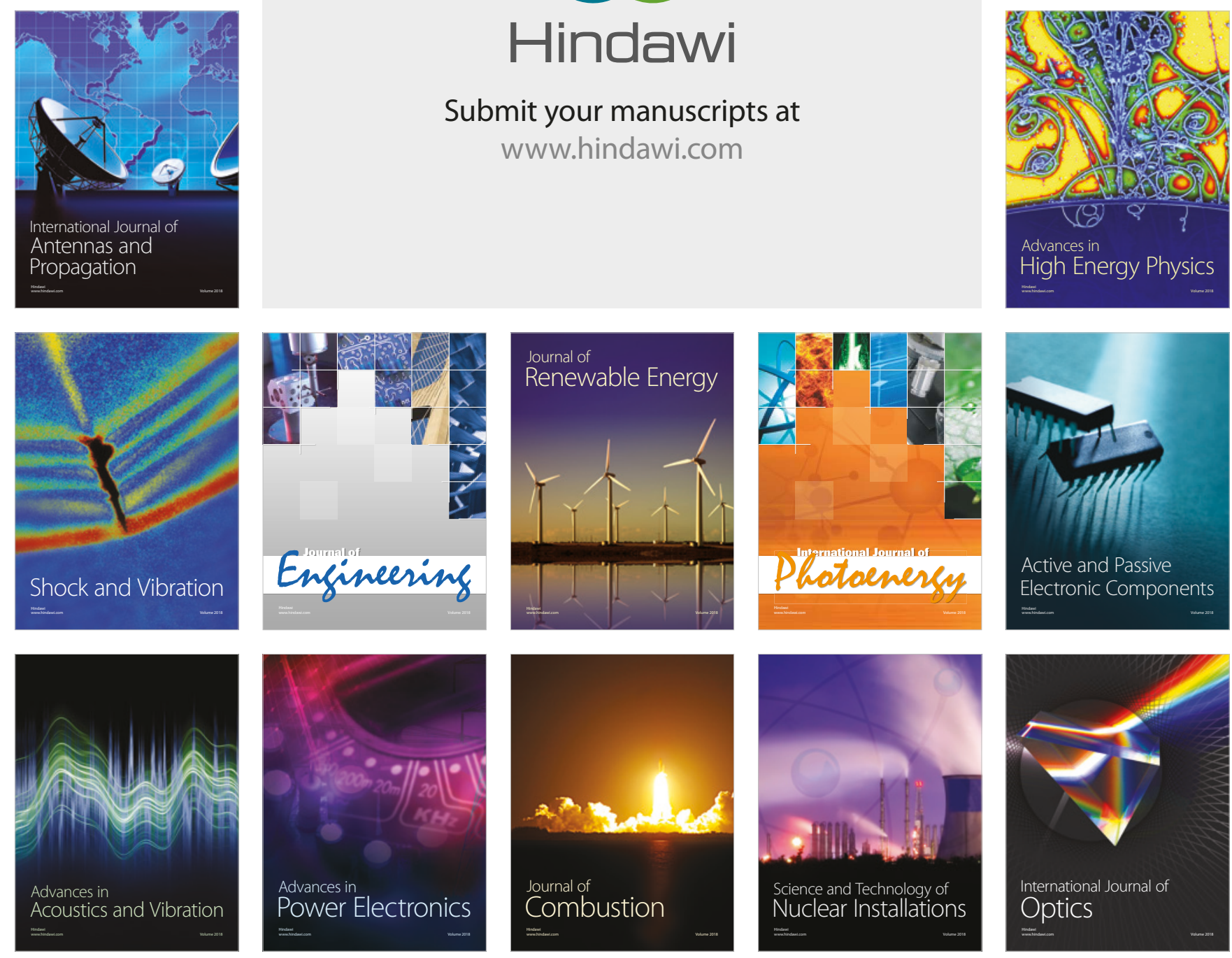